\title{
Analytical Stability Checks for Diaphragm Wall Trenches and Boreholes Supported by Polymer Solutions
}

\author{
Lesemann Henning ${ }^{1}$, Vogt Norbert ${ }^{2}$, Pulsfort Matthias ${ }^{3}$ \\ ${ }^{I}$ BAUER Spezialtiefbau GmbH, Technical Services, Schrobenhausen, Germany \\ ${ }^{2}$ Zentrum Geotechnik, Technische Universität München, Munich, Germany \\ ${ }^{3}$ Lehr- und Forschungsgebiet Geotechnik, Bergische Universität Wuppertal, Wuppertal, Germany

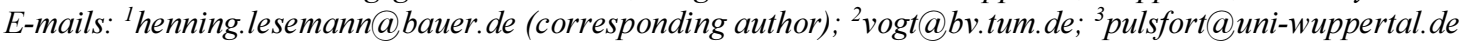

\begin{abstract}
Polymer solutions are increasingly used as supporting fluids for the temporary stabilization of diaphragm wall trenches and boreholes. However, analytical stability checks as they are common practice for geotechnical structures like retaining walls or slopes are rarely performed in these cases. Fundamental considerations for such stability checks will be presented here. For trenches and boreholes supported by bentonite suspensions analytical stability checks have proven themselves over a long time. Based on this concept, characteristics of hydraulic support by polymer solutions will be described in detail. The focus will be on the derivation of the time-dependent penetration length of a polymer solution into the surrounding foundation soil and of the hydraulic gradient over this penetration length which correlates with the efficiency of hydraulic support. Findings of other references dealing with three-dimensional failure body geometry, layered soils etc. may be combined with the approach in this paper as the hydraulic gradient around the trench or borehole is always required for such investigations. Unlike trial trenches or boreholes (typical in construction practice) analytical stability checks allow to evaluate relevant parameters for a specific application and to determine a level of safety for a given system. Such calculations should therefore complement trials on site.
\end{abstract}

Keywords: supporting fluid, polymer solution, diaphragm wall, bored pile, trench, borehole.

Conference topic: Design experiences and theoretical solutions.

\section{Introduction}

In worldwide bored pile or diaphragm wall projects polymer solutions have established themselves as an alternative to bentonite suspensions for the hydraulic support of boreholes and diaphragm wall trenches (Brown et al. 2002; Bustamante, Boato 2005; Heizmann et al. 2008). Polymer solutions are usually mixed on site by adding polymer powder to water. The main advantages are a simplified construction method (easier mixing and regeneration, reduced site equipment, more convenient consistency of excavated soil for disposal etc.) and often lower disposal costs for excess slurry. Consequently, polymer solutions offer economic chances despite relatively high material costs. However, approved analytical checks for borehole and trench stability as e.g. provided in German design standard DIN 4126 (2013) only consider bentonite suspensions and cannot be directly applied to fluids with no yield strength like most polymer solutions.

A research project at the Zentrum Geotechnik of the Technische Universität München (Germany) aimed to adapt the existing checks to polymer solutions. The project focused on investigating the flow behaviour in porous media of these fluids. A detailed documentation including further information concerning construction practice and environmental issues can be found in Lesemann (2010, in German). Steinhoff (1993), Majano, O'Neill (1993) and Kheng (1989) have provided further research reports on polymer supporting fluids. Steinhoff also investigated the flow behaviour and supporting mechanism while other reports mainly address influences on the bearing capacity of piles or sedimentation of soil particles within such fluids. This paper outlines the principles of analytical stability checks for polymer supported trenches and boreholes.

\section{Stability checks for trenches and boreholes}

\section{Trenches}

Stability checks for trenches supported by bentonite suspensions are well established since decades. Nevertheless, they will be briefly presented here as they serve as starting point for the following considerations regarding bentonite supported boreholes as well as polymer supported trenches and boreholes. DIN 4126 (2013) requires two types of stability checks: failure body check and single grains check.

The failure body check considers a failure body (Fig. 1) sliding on a shear plane with angle $\vartheta_{a}$. The gravitational force $G$ and a variable load $p$ act as driving forces while the body is stabilized by the shear force $Q$ on the shear plane $(\varphi=$ angle of friction $)$ and shear forces $T$ in the lateral faces. A reduction of the trench length $l_{t}$ increases the influence of the lateral shear forces $T$ and the level of safety. The vectorial difference between driving and stabilizing forces defines the horizontal earth pressure force $E_{a h}$. To prevent failure the supporting fluid must apply an effective force $S_{\text {effective }}>E_{a h}$. The supporting fluid with specific weight $\gamma_{F}$ opposes a total supporting force to the surrounding soil which is independent of the advancing penetration zone (Fig. 1) and 
equals the hydrostatic forces of the supporting fluid $S_{H}$ less those of the groundwater $W$ with specific weight $\gamma_{W}$. Since only a part of this supporting force is transferred within the relevant failure body, the effective supporting force $S_{\text {effective }}$ must be taken into account. In case of bentonite support a constant hydraulic gradient develops over the total area of penetration as soon as the penetration has stagnated due to the fluids' yield stress. $S_{\text {effective }}$ can then simply be calculated with the ratio between the area of penetration within the failure body $A_{1}$ and the total area of penetration $A_{1}+A_{2}$.

$$
S_{\text {effective }}=\left(S_{H}-W\right) \cdot \frac{A_{1}}{A_{1}+A_{2}}
$$

For supporting fluids without yield strength (like polymer solutions) there is no stagnation of the penetration. Penetration length and hydraulic gradient are timedependent functions. How these parameters may be calculated will be explained in the following sections. The presented approach may be combined with various approaches in other references dealing with failure body geometry, inclined ground surfaces, layered ground, etc. (e.g. Li et al. 2013a, 2013b).

The single grains check verifies that single grains or small groups of grains at the trench wall can be kept in place. These grains are pushed against the wall by the horizontal flow force $i \cdot \gamma_{F} \cdot V$. This flow force is a mass force which results from the hydraulic gradient $i$ within the volume $V$ of the considered grains. In case of a bentonite suspension with a yield strength this mass force is applied by static shear stresses after the penetration has stagnated. The horizontal flow force mobilizes an upward friction force which must be greater than the gravitational force of the grains (with buoyant specific weight under supporting fluid) to prevent sliding:

$$
\underbrace{\underbrace{i \cdot \gamma_{F} \cdot V}_{\text {flow force }} \cdot \tan \varphi}_{\text {friction force }} \geq \underbrace{\gamma^{\prime \prime} \cdot V}_{\text {weight force }},
$$

where: $\quad i=\frac{\Delta u}{l}=\frac{\Delta p / \gamma_{F}}{l}$.

In Eq. (2) $\Delta u[\mathrm{~m}]$ and $\Delta p\left[\mathrm{kN} / \mathrm{m}^{2}\right]$ are the potential difference and the pressure difference over the horizontal penetration length $l$ at a given depth of the trench. If the flow force is insufficient to retain the first grains at the wall, a gradual sliding of adjacent grains may result in a collapse of the trench.

\section{Boreholes}

For the failure body check of boreholes the earth pressure may e.g. be calculated considering a combined failure body after Walz, Hock (1987, Fig. 2). Alternatively, a fictive short trench can be assumed to perform a simplified check.

For bentonite suspensions after stagnation there is no difference in the single grains check between trenches and boreholes. Therefore, this type of check is frequently relevant for boreholes where the risk of a sliding failure body is low due to $3 \mathrm{D}$ effects.
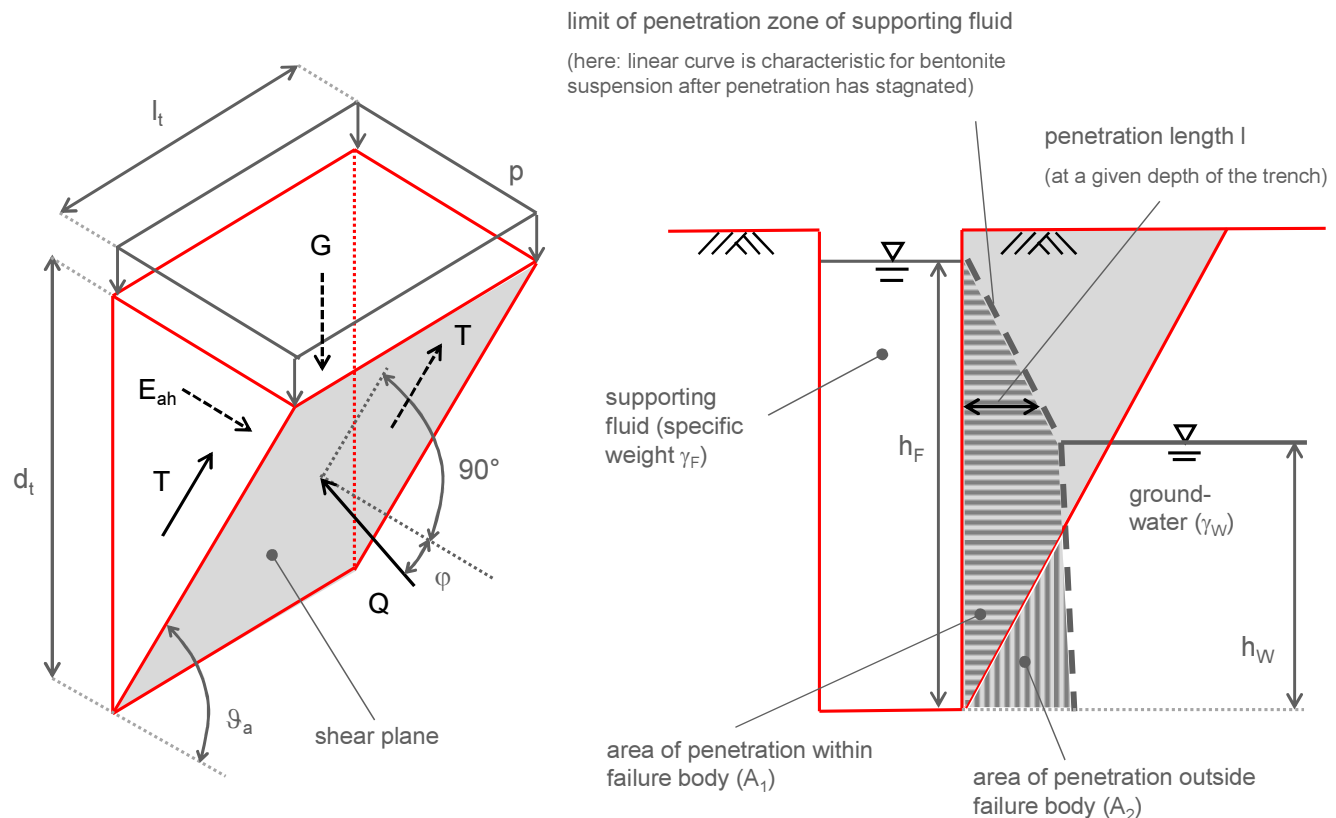

Fig. 1. Failure body model and penetration of bentonite suspension, adapted from DIN 4126 (2013) 

polymer solutions

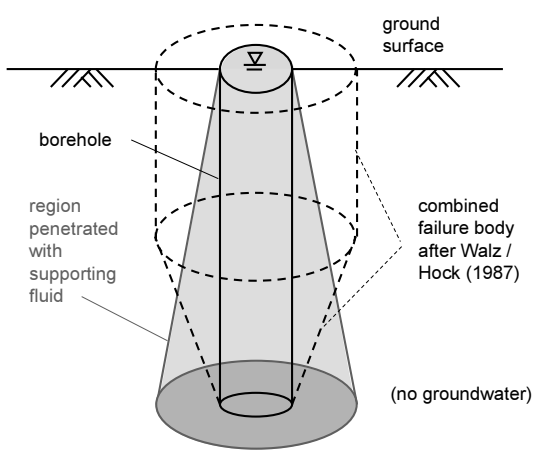

Fig. 2. Borehole stability, system with combined failure body after Walz, Hock (1987)

\section{Rheological properties and capillary flow of supporting fluids}

\section{Rheological properties}

The flow behaviour of supporting fluids depends on their rheological properties which can be characterized using rheological models. These are often described in shear rate vs. shear stress diagrams ("flow curves"). The viscosity of Newtonian fluids (e.g. water) is independent of the applied shear stress. Non-Newtonian fluids are generally divided into generalized Newtonian fluids (GNF) and other non-Newtonian fluids (e.g. fluids with viscoelastic properties) (Chhabra 2007). The only non-Newtonian attribute of GNF is a functional dependence between shear rate and viscosity:

$$
\eta=f(\dot{\gamma})
$$

The majority of GNF - and most polymer solutions regarded here - show a pseudo-plastic behavior. This refers to a sub-proportional relation of shear stress and shear rate, i.e. viscosity decreases with increasing shear rates. The rigid molecular chains of the polymers tend to align parallel to the flow direction at higher shear rates, thus reducing the resistance against shear deformation (Sorbie 1991).

The most common non-Newtonian model, the Ostwald-de Waele model (often simply "power law") (Balhoff 2005; Chhabra 2007), describes the pseudoplastic behavior by a power law with the parameters $K$ and $m$ (Table 1) which define the dependence between shear rate and viscosity. (Caution: The standard notation in Table 1 is incorrect regarding units if $\mathrm{m} \neq 1$. The following units are assumed: $m[-], K[\mathrm{~Pa} \cdot \mathrm{s}], \dot{\gamma}\left[\mathrm{s}^{-1}\right], \eta[\mathrm{Pa}$ - s] and $\tau[\mathrm{Pa}]$. This applies analogously to other models.) A disadvantage of the power law model is that viscosity tends to infinity as the shear rate approaches zero (for $m$ $<1$ ). A reasonable alternative to the power law is the Ellis model which limits the viscosity by an asymptotic value for low shear rates. This may help to obtain an acceptable representation over the relevant range of shear rates.

A plastic material behaves like a solid if exposed to low shear stress, but like a fluid if the shear stress exceeds a limit termed yield strength (DIN 1342-3, 2003). The flow curve beyond the yield strength may be linear (e.g. Bingham model) or non-linear (e.g. Herschel-Bulkley model, Table 1). Though not strictly correct, these materials are usually also denoted "fluids". Bentonite suspensions belong to this group of fluids.

Table 1. Rheological models and related equations for capillary flow

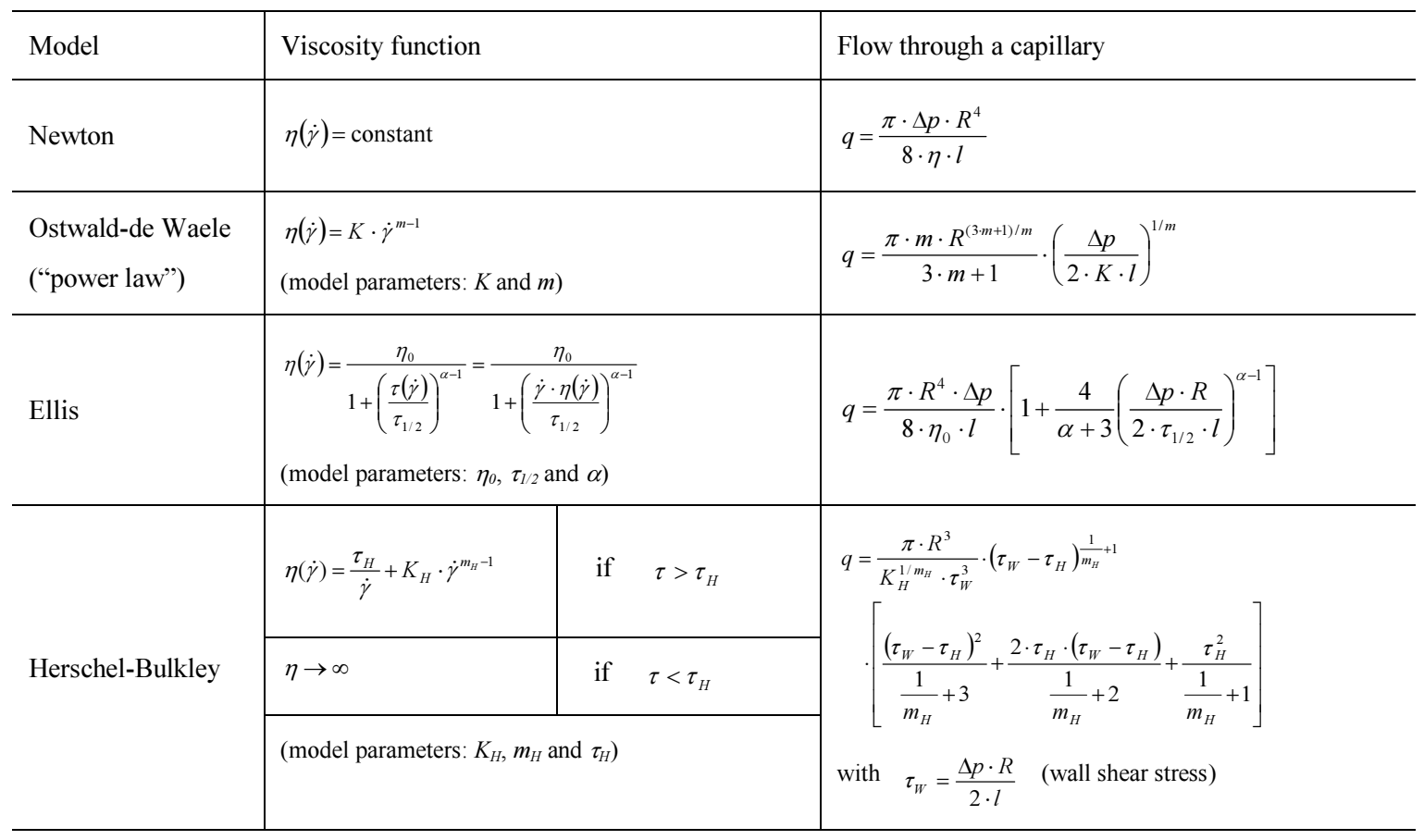




\section{Capillary flow}

In laminar capillary flow fluid particles move in concentric cylindrical layers orientated parallel to the capillary axis (Fig. 3). The layers transfer shear stresses among each other, so that the pressure difference $\Delta p$ over the capillary section with length $l$ finally dissipates at the capillary wall (where the outer fluid layer adheres). The shear stresses $\tau$ are defined by the equilibrium of forces between end planes and lateral area of a cylinder. They are a function of $r$ and independent of the fluids ' rheological properties.

$$
\pi \cdot r^{2} \cdot \Delta p=2 \pi \cdot r \cdot l \cdot \tau \Rightarrow \tau(r)=\frac{r \cdot \Delta p}{2 \cdot l} .
$$

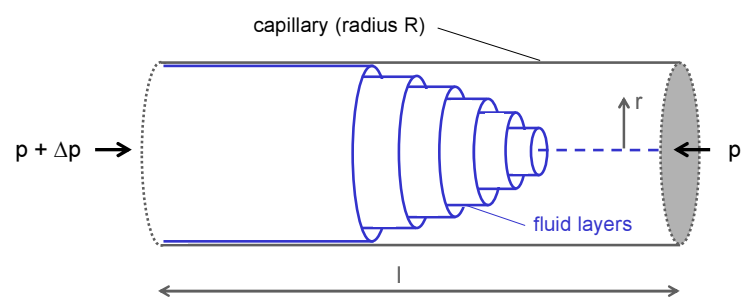

Fig. 3. Capillary flow, adapted from Darley, Gray (1988)

Radial velocity profile and flow rate through the capillary can be derived for a respective rheological model by inserting the viscosity function. For power law fluids with model parameters $K$ and $m$ the velocity profile $v(r)$ over the capillary cross section results in:

$v(r)=\left(\frac{\Delta p}{2 \cdot K \cdot l}\right)^{1 / m} \cdot \frac{m}{m+1} \cdot R^{(1+m) / m} \cdot\left[1-\left(\frac{r}{R}\right)^{(1+m) / m}\right]$.

And the flow rate $q$ (Sorbie 1991):

$$
q=\frac{\pi \cdot m \cdot R^{(3 \cdot m+1) / m}}{3 \cdot m+1} \cdot\left(\frac{\Delta p}{2 \cdot K \cdot l}\right)^{1 / m} .
$$

Eq. (6) corresponds to the well-known law of Hagen-Poiseuille for $m=1$ and $K=\eta$ (Newtonian fluid). Analogous equations can be deduced for all other models in Table 1 (Balhoff 2005; Chhabra 2007; Sorbie 1991).

\section{Calculation of the penetration length}

\section{Plastic fluids: penetration length within an infinite period of time}

The penetration of plastic fluids like bentonite suspensions into the foundation soil approaches a finite limit within an infinite period of time due to the existence of a yield strength $\tau_{\text {yield }}$. Therefore, a simplified stability check based on the maximum possible penetration length can be performed. This type of check is on the safe side as this maximum length may not be reached until the di- aphragm wall panel or bored pile is concreted. The stability checks implemented in DIN 4126 (2013) follow this concept (Fig. 1). The hydraulic gradient $i_{\text {stagnation }}$ at which stagnation occurs can be determined in a laboratory test or estimated with an empirical formula based on $\tau_{y i e l d}$ and the grain-size distribution of the soil to be supported.

\section{General approach: time-dependent penetration length}

In the general case (no yield strength, no stagnation of flow) the penetration length $l$ after a defined period of time (usually the period of time required from start of excavation until completion of concreting of the trench or pile) must be calculated. The equations in Table 1 for capillary flow can be adapted to quantitatively describe the flow of non-Newtonian fluids in porous media if the porous medium is specified using pore space models (Bear 1988). Examples for such models are shown in Figure 4.
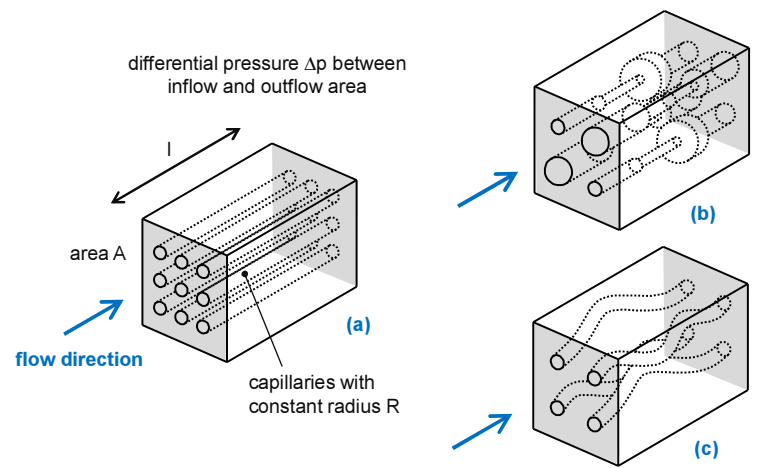

Fig. 4. Pore space models, adapted from Bear (1988)

The simplest pore space model consists of $N$ straight capillaries with constant radius $R$ (model (a) in Fig. 4). If porosity $n$ and flow rate $q$ of Newtonian fluids for a given hydraulic gradient shall be equal for model and represented porous medium, $N$ and $R$ can be derived.

Condition 1 (same porosity):

$$
N \cdot \pi \cdot R^{2}=n \cdot A(A=\text { flow area }),
$$

condition 2 (same flow rate for Newtonian fluids):

$$
N \cdot \frac{\pi \cdot \Delta u \cdot \gamma_{W} \cdot R^{4}}{8 \cdot \eta_{W} \cdot l}=k \cdot \frac{\Delta u}{l} \cdot A .
$$

The symbols $\gamma_{W}, \eta_{W}$ and $k$ represent the specific weight, viscosity and Darcy conductivity (in $[\mathrm{m} / \mathrm{s}]$ ) of water at $10{ }^{\circ} \mathrm{C}$. On the left-hand side of condition 2, $\Delta p$ has been replaced by $\Delta u \cdot \gamma_{W}$. This allows applying the equations in Table 1 to non-horizontal capillaries. Solving the system of equations gives:

$$
\begin{gathered}
N=\frac{n \cdot A}{\pi \cdot R^{2}}=\frac{n^{2} \cdot \gamma_{W}}{8 \cdot \pi \cdot \eta_{W} \cdot k} \cdot A ; \\
R=\sqrt{\frac{8 \cdot \eta_{W} \cdot k}{n \cdot \gamma_{W}}} .
\end{gathered}
$$


A reasonable variation of this model arranges one third of the capillaries in each Cartesian direction to allow flow in all directions (Sorbie 1991). As before, the porosity of the pore space model shall equal that of the porous medium. Therefore, the flow area $A$ in condition 1 must be replaced by $1 / 3 \cdot A$. Solving the modified set of equations yields:

$$
N_{3 D}=\frac{1 / 3 \cdot n \cdot A}{\pi \cdot R_{3 D}^{2}}=\frac{n^{2} \cdot \gamma_{W}}{72 \cdot \pi \cdot \eta_{W} \cdot k} \cdot A,
$$

(number of capillaries in one coordinate direction)

$$
R_{3 D}=\sqrt{\frac{24 \cdot \eta_{W} \cdot k}{n \cdot \gamma_{W}}} .
$$

It is important to keep in mind that $N_{3 D}$ and $R_{3 D}$ are merely used to calculate the flow rate $q$ as a non-linear function of the hydraulic gradient $\Delta u$. Though no nodes between capillaries of different orientation are explicitly modeled, the capillaries are supposed to communicate with each other. This allows e.g. flow in radial direction from a borehole as covered later.

Further variations of the capillary model are shown in Figure 4. Such enhanced models allow a more precise model since certain rheological fluid properties may only have an impact in combination with them. However, the mathematical descriptions get more complex and the soil parameters required to explicitly define such models are usually unavailable in practical applications.

\section{One-dimensional penetration (trench)}

Subsequently, the pore space model defined by $N_{3 D}$ and $R_{3 D}$ will be presumed. A closed form solution for the onedimensional, horizontal penetration length $l$ of a power law fluid as a function of time $t$ is derived. Vertical flow components can be neglected as proven in numerical simulations (Lesemann 2010): The pseudo-plastic fluid properties cause a very high viscosity in the vertical direction where hydraulic gradients are much smaller than in the horizontal direction, thus inducing a "pseudo-anisotropy" of the Darcy conductivity.

Within a time increment $d t$ the (incompressible) fluid volume flowing through the fictive capillaries must equal the filled pore volume according to the incremental penetration length $d l$. The flow rate $q$ through the area $A$ is the product of the flow rate through a single capillary and the number $N_{3 D}$ of capillaries normal to $A$.

$$
\begin{aligned}
& \underbrace{q(l) \cdot N_{3 D} \cdot d t}_{\text {fluid inflow }}=\frac{n^{2} \cdot \gamma_{W}}{72 \cdot \eta_{W} \cdot k} \cdot A \\
& \quad \frac{m \cdot R_{3 D}^{(3 \cdot m+1) / m}}{3 \cdot m+1} \cdot\left(\frac{\Delta u \cdot \gamma_{F}}{2 \cdot K \cdot l}\right)^{1 / m} \cdot d t=\underbrace{n \cdot A \cdot d l}_{\text {filled pore space }}
\end{aligned} .
$$

This differential equation can be solved by direct integration. After applying the initial condition $l(t=0)$ $=0$ to the solution the penetration length can be resolved:

$$
l=\left(\frac{(m+1) \cdot n \cdot \gamma_{W} \cdot R_{3 D}^{(3 \cdot m+1) / m}}{72 \cdot \eta_{W} \cdot k \cdot(3 \cdot m+1)} \cdot\left(\frac{\Delta u \cdot \gamma_{F}}{2 \cdot K}\right)^{1 / m} \cdot t\right)^{\frac{m}{m+1}}
$$

Derivations for other rheological models are complex, but equations analogous to Eq. (13) may still be integrated numerically if a closed solution cannot be found.

To determine reliable rheological parameters of a fluid in the lab (as discussed later) the relevant range of shear rates must be estimated. As the shear rates vary over the cross section of a capillary, apparent viscosity $\eta_{a p p}$ and apparent shear rate $\dot{\gamma}_{\text {app }}$ are used as reference values. The apparent viscosity is the viscosity of a fictive Newtonian fluid that experiences the same flow rate as the actual non-Newtonian fluid given the same hydraulic gradient.

$$
\begin{gathered}
q_{\text {Newtonian }}=\frac{\pi \cdot \Delta u \cdot \gamma_{F} \cdot R_{3 D}^{4}}{8 \cdot \eta_{a p p} \cdot l}=q_{\text {non-Newtonian }} \\
\Rightarrow \eta_{\text {app }}=\frac{\frac{\pi \cdot \Delta u \cdot \gamma_{F} \cdot R_{3 D}^{4}}{8 \cdot l}}{q_{\text {non-Newtonian }}}
\end{gathered}
$$

After substituting the equations of Table 1 in Eq. (15) apparent viscosity and apparent shear rate of a power law fluid can be obtained as follows:

$$
\begin{gathered}
\eta_{a p p}=\frac{\frac{\pi \cdot \Delta u \cdot \gamma_{F} \cdot R_{3 D}^{4}}{8 \cdot l}}{\frac{\pi \cdot m \cdot R_{3 D}^{(3 \cdot m+1) / m}}{3 \cdot m+1} \cdot\left(\frac{\Delta u \cdot \gamma_{F}}{2 \cdot K \cdot l}\right)^{1 / m}} ; \\
\dot{\gamma}_{a p p}=\dot{\gamma}\left(\eta_{a p p}\right)=\left(\frac{\eta_{a p p}}{K}\right)^{\frac{1}{m-1}} .
\end{gathered}
$$

The actual shear rates of the non-Newtonian fluid in the capillary model (and in the real porous medium) are both higher and lower than the apparent shear rate.

\section{Radial-symmetric penetration (borehole)}

For continuity reasons the flow rate $q$ passing through circumferential areas of any distance to the borehole must be constant at a given time. However, this does not apply to flow velocity and hydraulic gradient in radial direction $d u / d r$. Symbols are explained in Figure 5 .

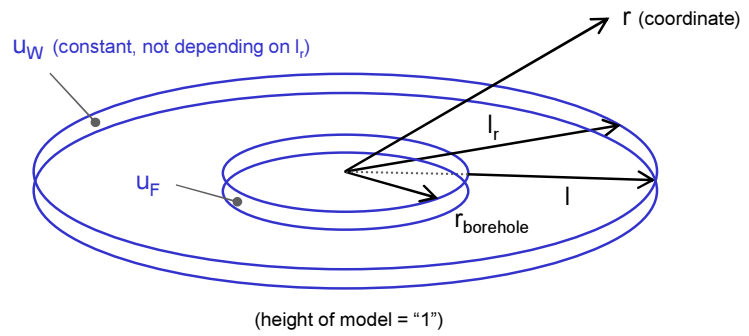

Fig. 5. Penetration from borehole (radial symmetry) 
The actual penetration length into the formation will still be designated with $l$. The penetration length $l_{r}$ measured from the borehole axis is introduced additionally. Analogous to the one-dimensional case the flow rate through the circumferential area $2 \cdot \pi \cdot \mathrm{r}$ (with height " 1 ") is determined by:

$$
q=\frac{n^{2} \cdot \gamma_{W}}{72 \cdot \eta_{W} \cdot k} \cdot \frac{m \cdot R_{3 D}^{(3 \cdot m+1) / m}}{3 \cdot m+1} \cdot\left(\frac{-\frac{d u}{d r} \cdot \gamma_{F}}{2 \cdot K}\right)^{1 / m} \cdot 2 \cdot \pi \cdot r
$$

After solving this differential equation by separating the variables and substitution of boundary condition $u\left(r=r_{\text {borehole }}\right)=u_{F}$ the potential $u$ can be determined as a function of $r$. (A more detailed derivation is provided in Lesemann (2010)).

$$
\begin{gathered}
u=u_{F}-\frac{q^{m} \cdot \frac{2 \cdot K}{\gamma_{F}}}{\left(\frac{n^{2} \cdot \gamma_{W}}{36 \cdot \eta_{W} \cdot k} \cdot \frac{\pi \cdot m}{3 \cdot m+1}\right)^{m} \cdot R_{3 D}^{3 \cdot m+1}} \\
\cdot \frac{r^{1-m}-r_{\text {borehole }}^{1-m}}{1-m}
\end{gathered}
$$

With the boundary condition $u\left(r=l_{r}\right)=u_{W}$ the flow rate can be calculated $\left(\Delta u=u_{F}-u_{W}\right)$ :

$$
\begin{gathered}
q=\left(\frac{\Delta u \cdot \gamma_{F}}{2 \cdot K}\right)^{1 / m} \cdot \frac{n^{2} \cdot \gamma_{W}}{36 \cdot \eta_{W} \cdot k} \cdot \frac{\pi \cdot m}{3 \cdot m+1} \\
\cdot R_{3 D}^{(3 \cdot m+1) / m} \cdot\left(\frac{1-m}{l_{r}^{1-m}-r_{\text {borehole }}^{1-m}}\right)^{1 / m}
\end{gathered}
$$

As in the one-dimensional case, the penetrating fluid volume must equal the additionally filled pore volume:

$$
\begin{gathered}
q\left(l_{r}\right) \cdot d t=n \cdot 2 \cdot \pi \cdot l_{r} \cdot d l_{r} ; \\
\Rightarrow \quad\left(\frac{\Delta u \cdot \gamma_{F}}{2 \cdot K}\right)^{1 / m} \cdot \frac{n \cdot \gamma_{W}}{72 \cdot \eta_{W} \cdot k} \cdot \frac{m}{3 \cdot m+1} \cdot R_{3 D}^{(3 \cdot m+1) / m} \\
\cdot(1-m)^{1 / m} \cdot \int d t=\int\left(l_{r}^{1-m}-r_{\text {borehole }}^{1-m}\right)^{1 / m} \cdot l_{r} \cdot d l_{r}
\end{gathered}
$$

The variables of this differential equation can be separated, but the resulting integral on the right-hand side of the equation cannot be solved analytically. It is nevertheless possible to obtain individual solutions by means of numerical integration in the intervals from 0 to $t$ and $r_{\text {borehole }}$ to $l_{r}$ respectively.

\section{Hydraulic gradient function for boreholes}

For one-dimensional flow from an infinitely long trench the flow velocity of an incompressible fluid at a given depth must, for continuity reasons, be constant over the penetration length (if vertical flow components are neglected). Accordingly, the hydraulic gradient is constant along this length regardless of the rheological properties of the fluid. In the radial-symmetric case of a borehole the hydraulic gradient is only constant if the penetration has stagnated (i.e. bentonite support), whereas polymer solutions generally cause a non-linear potential function. Inserting Eq. (20) in Eq. (19) the potential $u$ and the hydraulic gradient $d u / d r$ of a penetrating power law fluid may be determined as a function of $r\left(r_{\text {borehole }} \leq r \leq l_{r}\right)$ :

$$
\begin{gathered}
u(r)=u_{F}-\frac{r^{1-m}-r_{\text {borehole }}^{1-m}}{l_{r}^{1-m}-r_{\text {borehole }}^{1-m}} \cdot \Delta u ; \\
\frac{d u}{d r}=\frac{m-1}{l_{r}^{1-m}-r_{\text {borehole }}^{1-m}} \cdot \frac{\Delta u}{r^{m}} .
\end{gathered}
$$

The maximum hydraulic gradient prevails at the borehole wall and can be calculated with Eq. (24) and $r=r_{\text {borehole }}$. This has a positive effect on hydraulic support. Applying the average hydraulic gradient (along the penetration length) in the failure body check or single grains check would be very conservative.

\section{Calculation of the effective supporting force for the fail- ure body check}

If a bentonite suspension is used as supporting fluid and the stability checks performed for the maximum penetration length after an infinite period of time the effective supporting force for the failure body check could be calculated by multiplying the total hydraulic supporting force with the area ratio between the area of penetration within the failure body and the total area of penetration (Eq. (1)). This calculation method is only valid, if the hydraulic gradient is constant within the entire penetration zone (i.e. after stagnation of penetration). In case of hydraulic support with a pseudo-plastic fluid without yield strength the penetration length increases sub-proportionally with depth $z$. As the potential difference $\Delta u$ is a linear function of $z$, the hydraulic gradient $i=\Delta u / l$ increases with the depth (Fig. 6).

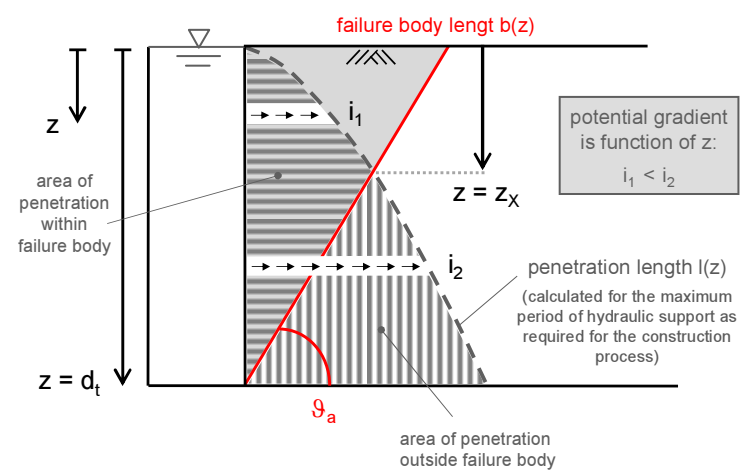

Fig. 6. Penetration of polymer solution and calculation of effective hydraulic supporting force

In general, the effective supporting force must therefore be determined by integration of the flow forces over the area of penetration within the failure body. The calculation for the trench shown in Figure 6 would be: 

polymer solutions

$$
\begin{aligned}
S_{\text {effective }} & =\int_{A} i(z) \cdot \gamma_{F} \cdot d A= \\
& \int_{0}^{z_{X}} \int_{0}^{l(z)} \frac{\Delta u(z)}{l(z)} \cdot \gamma_{F} \cdot d x \cdot d z+ \\
& \int_{z_{X}}^{d_{t}} \int_{0}^{b(z)} \frac{\Delta u(z)}{l(z)} \cdot \gamma_{F} \cdot d x \cdot d z= \\
& \int_{0}^{z_{X}} \Delta u(z) \cdot \gamma_{F} \cdot d z+\int_{z_{X}}^{d_{t}} \Delta u(z) \cdot \frac{b(z)}{l(z)} \cdot \gamma_{F} \cdot d z .
\end{aligned}
$$

The calculation for the radial-symmetric case (borehole) is analogous, but with a hydraulic gradient varying in radial direction as well.

\section{Advanced characteristics of hydraulic support}

Some advanced characteristics of supporting fluid penetration are discussed in Lesemann (2010). Among them are: visco-elastic fluid properties, the possible formation of a pressure-transferring polymer membrane at the borehole or trench wall, partially-saturated conditions in case of polymer penetration above groundwater level and a decreasing polymer concentration of the supporting fluid due to polymer adsorption in the formation.

Of particular practical importance is the reduction of soil conductivity near the borehole or trench caused by the filtration of soil particles (colmatation) which are incorporated into the supporting fluid during the drilling process. This may significantly reduce fluid loss to the formation and improve hydraulic support efficiency as this causes an increase of hydraulic gradients near the wall. Sometimes materials like mineral fibres, nutshells or highly swellable polymers ("superadsorbers") are added to the supporting fluid on purpose. However, to include such filtration effects in the calculation of penetration length and hydraulic gradients would be very complex. The content and size of particles in the drilling fluid depends on the grainsize distributions of the different soil layers to be excavated, of fluid parameters like viscosity and specific density (settlement of particles within the borehole) and of the operating parameters of the drilling rig and regeneration plant. If filtration effects are ignored the calculations will be more or less conservative.

\section{Determination of model parameters}

The required rheological parameters (e.g. power law parameters $K$ and $m$ ) for the calculation of the penetration $l(t)$ may be determined in two ways:

- Determination on the basis of measured flow curves (e.g. with rotational viscosimeter).

- Determination by back-calculation of 1D flow tests ("in-situ parameters").

Using the first method, it is very important to determine the flow curve in a range of shear rates which is representative for the flow process to be modeled (cf. Eq. (17)). Depending on the specific application this can eventually only be met with special viscosimeters for very small shear rates.

The execution of 1D flow tests (Fig. 7) is recommended (Lesemann 2010). The test allows measuring of the time-dependent penetration of a polymer solution into a soil column under defined conditions.

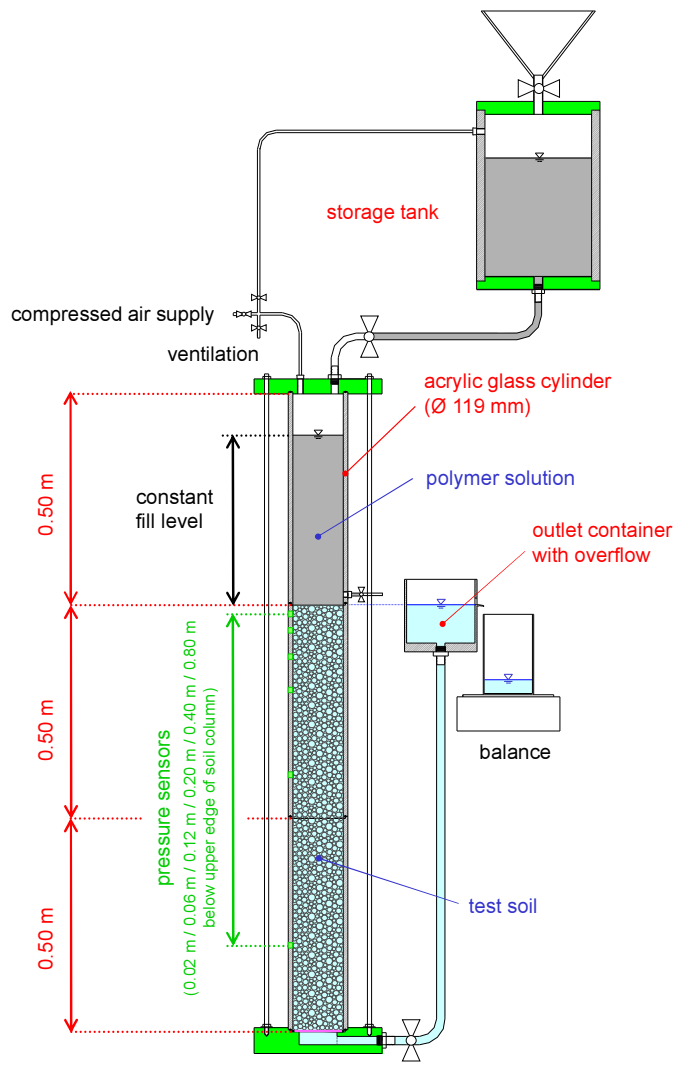

Fig. 7. Sketch of test rig for 1D flow tests

With 1D flow tests the required model parameters can be determined by curve-fitting. These "in-situ parameters" implicitly include correction factors for the insufficiencies of the analytical model (e.g. simplification of the porous medium by a pore space model). With parameters determined in this way the penetration curves for changed boundary conditions (soil type or potential difference) could be predicted rather precisely in a large series of tests. At the same time, this can be regarded an implicit validation of the presented equations.

Finally, this method was successfully used to predict fluid loss and borehole stability for full-scale field tests (Lesemann 2010). Here, "in-situ parameters" had been investigated in 1D flow tests for a single reference soil type in combination with all polymer products and concentrations to be used in the field.

\section{Conclusions}

In technical and economic terms, polymer solutions are an attractive alternative to bentonite suspensions 

polymer solutions

for the hydraulic support of trenches and boreholes. Their flow behavior differs significantly due to the non-existent yield strength. Well established stability checks for bentonite support may nevertheless be adapted if the time-dependent penetration process is taken into account. The flow of polymer solutions can be described analytically using pore space models. The required model parameters may be determined with viscosimeter measurements or - preferably - on the basis of $1 \mathrm{D}$ flow tests.

It is generally recommended to perform analytical stability checks. Compared to trial trenches or boreholes there are a number of advantages: quantitative evaluation of the level of safety (instead of a simple "failure/no failure"), sensitivity analysis for the parameters of the mechanical system (shear strength of soil, groundwater level, geometry of trench or borehole, variable loads etc.). Ideally, analytical checks serve to optimize the planning of complementary trial excavations.

Future research should aim to find a rough quantitative estimation for the positive filtration effects (reduction of fluid loss, improved support efficiency).

Finally, apart from borehole or trench stability, various other aspects must be paid attention to when polymer support is considered: possible impacts on concrete pour, regeneration of used slurry, environmental compatibility etc. (cf. Lesemann 2010).

\section{Funding}

The research project was supported by the research program "Zukunft Bau" of the German Federal Office for Building and Regional Planning [grant number Z $6-10.08 .18 .7-$ 07.11 / II 2 - F20-07-12] as well as by (former) Bilfinger Berger AG and (former) Süd-Chemie AG.

\section{Disclosure statement}

Authors do not have any competing financial, professional, or personal interests from other parties.

\section{References}

Balhoff, M. 2005. Modelling the flow of non-newtonian fluids in packed beds at the porescale: Dissertation. Louisiana State University.

Bear, J. 1988. Dynamics of fluids in porous media. New York: Dover Publ.
Brown, D.; Muchard, M.; Khouri, B. 2002. The effect of drilling fluid on axial capacity, Cape Fear River, NC, in $27^{\text {th }}$ Annual Conference of the Deep Foundations Institute, 911 October 2002, San Diego, CA.

Bustamante, M.; Boato, R. 2005. Les polymères: Application au forage des pieux de grands diamètres, in Proc. $16^{\text {th }}$ ICSMGE, 12-16 September 2005, Osaka.

Chhabra, R. P. 2007. Bubbles, drops and particles in non-Newtonian fluids. Boca Raton: Taylor \& Francis Group.

Darley, H. C. H.; Gray, G. R. 1988. Composition and properties of drilling and completion fluids. $5^{\text {th }}$ ed. Houston: Gulf Publishing Company.

DIN 1342-3. Viscosity - part 3: non-newtonian liquids. Beuth Verlag, 2003.

DIN 4126. Stability analysis of diaphragm walls. Beuth Verlag, 2013.

Heizmann, A., et al. 2008. Gründungsarbeiten des Golden Ears Projektes in Vancouver, Bohrpfahlherstellung in ungewohnten Dimensionen. Baugrundtagung, Dortmund.

Kheng, H. Y. 1989. Rheological and Physico-Chemical Properties of Palygorskite and Anionic Polyacrylamide Polymer Slurries used in Drilled Shaft Construction. Ph. D. Dissertation, University of Florida, Gainsville.

Lesemann, H. 2010. Anwendung polymerer Stützflüssigkeiten bei der Herstellung von Bohrpfählen und Schlitzwänden, Dissertation, Zentrum Geotechnik, Technische Universität München.

Li, Y.-C.; Pan, Q.; Chen, Y.-M. 2013a. Stability of slurry trenches with inclined ground surface, Journal of Geotechnical and Geoenvironmental Engineering 139(9): $1617-1619$ http://dx.doi.org/10.1061/(ASCE)GT.1943-5606.0000868

Li, Y.-C.; Pan, Q.; Cleall, P. J.; Chen, Y.-M.; Ke, H. 2013 b. Stability analysis of slurry trenches in similar layered soils, Journal of Geotechnical and Geoenvironmental Engineering 139(12): 2104-2109. http://dx.doi.org/10.1061/(ASCE)GT.1943-5606.0000958

Majano, R. E.; O’Neill, M. W. 1993. Effect of mineral and polymer slurries on perimeter load transfer in drilled shafts. University of Houston.

Sorbie, K. S. 1991. Polymer-improved oil recovery. Glasgow, London: Blackie and Son Ltd. http://dx.doi.org/10.1007/978-94-011-3044-8

Steinhoff, J. 1993. Standsicherheitsbetrachtungen für polymergestützte Erdwände: Dissertation. Bergische Universität Gesamthochschule Wuppertal, LuFG Geotechnik, Bericht - Nr. 13.

Walz, B.; Hock, K. 1987. Berechnung des räumlich aktiven Erddrucks mit der modifizierten Ele-mentscheibentheorie. Bergische Universität Gesamthochschule Wuppertal, LuFG Geotechnik, Bericht - Nr. 6 . 